International Journal of Modern Physics D Vol. 26, No. 14 (2017) 1799001 (16 pages)

(C) World Scientific Publishing Company

DOI: $10.1142 / \mathrm{S} 0218271817990012$

\title{
Cumulative author index
}

Abbasvandi, N., see Soleimani

Abdujabbarov, A., Toshmatov, B., Schee, J., Stuchlík, Z. \& Ahmedov, B., Gravitational lensing by regular black holes surrounded by plasma

Abdujabbarov, A., Toshmatov, B., Stuchlík, Z. \& Ahmedov, B., Shadow of the rotating black hole with quintessential energy in the presence of plasma

Abebe, A., Dunsby, P. K. S. \& Solomons, D., Integrability conditions of quasiNewtonian cosmologies in modified gravity

Adak, D. \& Ali, A., Bounce and collapse in the slotheonic universe

Adler, S. L., Implications of a frame dependent gravitational effective action for perturbations on the Robertson-Walker metric

Agullo, I., del Rio, A. \& Navarro-Salas, J., Gravity and handedness of photons

Ahluwalia, D. V., Reflections of the observer and the observed in quantum gravity

Ahmed, S., see Hussain

Ahmedov, B., see Abdujabbarov

Ahmedov, B., see Abdujabbarov

Aimuratov, Y., see Rueda

Aimuratov, Y., see Ruffini

Al-Badawi, A., Owaidat, M. Q. \& Tarawneh, S., The geodesics structure of Schwarzschild black hole

$\mathbf{2 6}(2017) 1750043$
$\mathbf{2 6}(2017) 1741011$

26 (2017) 1750051

26 (2017) 1750054

26 (2017) 1750089

26 (2017) 1750159

26 (2017) 1742001

26 (2017) 1743001

26 (2017) 1750155

26 (2017) 1741011

26 (2017) 1750051

26 (2017) 1730016

26 (2017) 1730019 immersed in an electromagnetic universe

Al-Jamel, A., see Maurya

Alhamzawi, A., Observing the shadow of modified gravity black hole

Ali, A., see Adak

Amelino-Camelia, G., Barcaroli, L., D'Amico, G., Loret, N. \& Rosati, G., Quantum-gravity-induced dual lensing and IceCube neutrinos

Amen, S. N. H., see Mazharimousavi

Andersson, N., see Graber

Antoniadis, I. \& Cotsakis, S., Infinity in string cosmology: A review through open problems

Aoki, A. \& Soda, J., Detecting ultralight axion dark matter wind with laser interferometers

Argüelles, C. R., see Mavromatos

Arsenadze, G. \& Osmanov, $\mathrm{Z}$., Particles on the rotating channels in the wormhole metrics

Arzano, M. \& Calcagni, G., Entanglement entropy, scale-dependent dimensions and the origin of gravity

Assimos, T. S., Pereira, A. D., Santos, T. R. S., Sobreiro, R. F., Tomaz, A. A. \& Otoya, V. J. V., From $S L(5, \mathbb{R})$ Yang-Mills theory to induced gravity

Azhar, N., see Jawad

Azri, H. \& Bounames, A., Cosmological consequences of a variable cosmological constant model
26 (2017) 1750169

26(2017) 1750002

26 (2017) 1750156

26 (2017) 1750089

26 (2017) 1750076

26 (2017) 1750158

26 (2017) 1730015

26 (2017) 1730009

$26(2017) 1750063$

$26(2017) 1730007$

26 (2017) 1750153

26 (2017) 1743030

26 (2017) 1750087

26 (2017) 1750040

26 (2017) 1750060 
Baccetti, V., Mann, R. B. \& Terno, D. R., Do event horizons exist?

Bakhtadze, G. G., Berezhiani, V. I. \& Osmanov, Z., Centrifugal acceleration in the isotropic photon field

Ballardini, M., see Masi

Bamba, K., see Sepehri

Bandyopadhyay, P., see Chakravarty

Banerjee, $\mathrm{K}$, see Kunkolienkar

Banerjee, S., see Bhar

Banik, S., see Lenka

Bao, N., Carroll, S. M. \& Singh, A., The Hilbert space of quantum gravity is locally finitedimensional

Barcaroli, L., see AmelinoCamelia

Barreto, W., de Oliveira, H. P. \& Rodrigues, E. L., Nonlinear interaction between electromagnetic and gravitational waves: An appraisal

Barreto, W., de Oliveira, H. P. \& Rodriguez-Mueller, B., A (gravitational) toy story

Barrow, J. D. \& Ganguly, C., The shape of bouncing universes

Basilakos, S., see Kamali

Batool, M. \& Hussain, I., Null and timelike geodesics of the Schwarzschild black hole with string cloud background

Becerra, L., see Rueda

Becerra, L., see Ruffini

Belinchón, J. A., Harko, T. \& Mak, M. K., Exact scalartensor cosmological models

Belotsky, K. M., Kirillov, A. A., Nazarova, N. O. \& Rubin, S. G., Reionization effect enhancement due to primordial black holes
26 (2017) 1743008

26 (2017) 1750135

26 (2017) 1750041

26 (2017) 1750094

26 (2017) 1750122

26 (2017) 1750143

26 (2017) 1750078

26 (2017) 1750127

26 (2017) 1743013

26 (2017) 1750076

26 (2017) 1743017

26 (2017) 1750096

26 (2017) 1743016

26 (2017) 1743023

26 (2017) 1741005

26 (2017) 1730016

26 (2017) 1730019

26 (2017) 1750073

26 (2017) 1750102
Bendjoudi, A. \& Mebarki, N., The quantum tetrahedron and the length spectrum

Benisty, D. \& Guendelman, E. I., Unified DE-DM with diffusive interactions scenario from scalar fields

Berezhiani, V. I., see Bakhtadze

Bessada, D. F. A., see Tamayo

Bezerra, V. B., see Graça

Bhar, P. \& Govender, M., Anisotropic charged compact star of embedding class I

Bhar, P., Singh, K. N. \& Manna, T., A new class of relativistic model of compact stars of embedding class I

Bhar, P., Singh, Ksh. N., Rahaman, F., Pant, N. \& Banerjee, S., A charged anisotropic well-behaved Adler-Finch-Skea solution satisfying Karmarkar condition

Bhatia, A. S. \& Sur, S., Dynamical system analysis of dark energy models in scalar coupled MetricTorsion theories

26 (2017) 1750044

26 (2017) 1743021

26 (2017) 1750135

26 (2017) 1750093

26 (2017) 1750113

26 (2017) 1750053

26 (2017) 1750090

26 (2017) 1750078

Bhatt, J. R. \& George, M., Electromagnetic instability induced by neutrino interaction

Bhatti, M. Z. \& Yousaf, Z., Gravitational collapse and dark universe with LTB geometry

Bianco, C. L., see Rueda

Bianco, C. L., see Ruffini

Bogadi, R. S., see Govender

Bolejko, K. \& Korzyński, M., Inhomogeneous cosmology and backreaction: Current status and future prospects

Bombaci, I. \& Logoteta, D., Quark deconfinement in
26 (2017) 1750045

26 (2017) 1730016

26 (2017) 1730019

26 (2017) 1750065

26 (2017) 1730011 
neutron stars and astrophysical implications

Borkar, M. S. \& Gayakwad, P. V., LRS Bianchi type I magnetized cosmological model with perfect fluid and with quintessence, Chaplygin gas dark energy in bimetric theory of gravitation

Bounames, A., see Azri

Bozza, V., Gravitational lensing by black holes and their alternatives

Brandenberger, R. H., Cai, Y.-F., Wan, Y. \& Zhang, $\mathrm{X}$., Nonsingular cosmology from an unstable Higgs field

Brandenberger, R., Initial conditions for inflation A short review

Bretón, N., Clark, T. \& Fernando, S., Quasinormal modes and absorption cross-sections of BornInfeld-de Sitter black holes

Brevik, I., Grøn, Ø., de Haro, J., Odintsov, S. D. \& Saridakis, E. N., Viscous cosmology for early- and latetime universe

Brihaye, Y., Delsate, T., Sawado, N. \& Yoshii, H., Dirac equation for spherically symmetric $\mathrm{AdS}_{5}$ spacetime and application to a boson star in EGB gravity

Brinkerink, C., see Goddi

Bronzwaer, T., see Goddi

Buchel, A., Universality of small black hole instability in AdS/CFT

Bueno, P. \& Cano, P. A., Universally stable black holes

Buettner, D. J., see Morley

Burinskii, A., Weakness of gravity as illusion which hides true path to unification of gravity with particle physics

Cai, Y.-F., see Brandenberger
26 (2017) 1730004

26 (2017) 1750061

26 (2017) 1750060

26 (2017) 1741013

26 (2017) 1740006

26 (2017) 1740002

26 (2017) 1750112

26 (2017) 1730024

26 (2017) 1750086

26 (2017) 1730001

26 (2017) 1730001

26 (2017) 1750140

26 (2017) 1743024

26 (2017) 1750069

26 (2017) 1743022

26 (2017) 1740006
Calcagni, G., see Arzano

Caldwell, R. R., Devulder, C. \& Maksimova, N. A., Gravitational wave-gauge field dynamics

Cannizzo, J. K., see Gehrels

Cano, P. A., see Bueno

Capozziello, S., see Sepehri

Carroll, S. M., see Bao

Castellanos, E., EscamillaRivera, C., Lämmerzahl, C. \& Macías, A., Scalar field as a BoseEinstein condensate in a Schwarzschild-de Sitter spacetime

Chakrabarti, S., see Wahlang

Chakravarty, G. K., Mohanty, S. \& Lambiase, G., Testing theories of gravity and supergravity with inflation and observations of the cosmic microwave background

26 (2017) 1743030

26 (2017) 1742005

26 (2017) 1730003

26 (2017) 1743024

26 (2017) 1750094

26 (2017) 1743013

26 (2017) 1750032

26 (2017) 1750160

26 (2017) 1730023

Chakravarty, N., Mullick, L. \& Bandyopadhyay, P., Fermions, loop quantum gravity and geometry

Chan, M. H., Analytic expressions for the dark matter-baryon relations

Char, P., see Lenka

Chattopadhyay, S., Modified Chaplygin gas equation of state on viscous dissipative extended holographic Ricci dark energy and the cosmological consequences

Chaturvedi, P., Singh, N. K. \& Singh, D. V., ReissnerNordstrom metric in unimodular theory of gravity

Chen, G.-R., see Zhou

Chen, T., see Peng

Chen, X., Namjoo, M. H. \& Wang, Y., Probing the primordial universe using massive fields

Cherubini, C., see Rueda

Chilambwe, B., see Dadhich

Ciufolini, I., see Gurzadyan

Clark, T., see Bretón

Congedo, G., see De Marchi
26 (2017) 1750118

26 (2017) 1750127

26 (2017) 1750042

26 (2017) 1750082

26 (2017) 1750062

26 (2017) 1750046

26 (2017) 1740004

26 (2017) 1730016

26 (2017) 1750056

26 (2017) 1741020

26(2017) 1750112

26 (2017) 1741021 
Congedo, G., Detection principle of gravitational wave detectors

Conti, E. \& Sartori, G., On the coherent emission of radio frequency radiation from high energy particle showers

Cook, S. A., Low valence Killing spinors in Gödel's Universe

Cooperstock, F. I., Gravitational quanta and unification

Corrêa Silva, E. V., see Monerat

Corrêa Silva, E. V., see Oliveira-Neto

Cotsakis, S., see Antoniadis

Coumbe, D. N., Quantum gravity without vacuum dispersion

Cremaschini, C. \& Stuchlík, Z., Carter constant induced mechanism for generation of anisotropic kinetic equilibria in collisionless $N$-body systems

Dadhich, N., Hansraj, S. \& Chilambwe, B., Compact objects in pure Lovelock theory

Daghigh, M., see Sefiedgar

D'Amico, G., see AmelinoCamelia

Darabi, F., see Setare

Davelaar, J. R. J., see Goddi

Dayanandan, B., see Maurya

de Almeida, U. B., see Rueda

de Haro, J., see Brevik

de la Macorra, A., see Quintanar

De Laurentis, M., see Goddi

De Marchi, F. \& Congedo, G., Space tests of the strong equivalence principle: BepiColombo and the Sun-Earth Lagrangian points opportunity

de Oliveira, H. P., see Barreto

de Oliveira, H. P., see Barreto

De Paolis, F., see Giordano
De Paolis, F., see Nucita

Deane, R., see Goddi

26 (2017) 1741022

del Rio, A., see Agullo

Delsate, T., see Brihaye

Dent, J. B., Easson, D. A., Kephart, T. W. \& White, S. C., Stability aspects of

26 (2017) 1750083

26 (2017) 1750147

26 (2017) 1743029

26 (2017) 1750022

26 (2017) 1750011

26 (2017) 1730009

26 (2017) 1750119

26 (2017) 1750001

26 (2017) 1750056

26 (2017) 1750139

26 (2017) 1750076

26 (2017) 1750101

26 (2017) 1730001

26 (2017) 1750002

26 (2017) 1730016

26 (2017) 1730024

26 (2017) 1750004

26 (2017) 1730001

26 (2017) 1741021

26 (2017) 1743017

26 (2017) 1750096

26 (2017) 1741009 wormholes in $R^{2}$ gravity

Deriglazov, A. A. \& Ramírez, W. G., Mathisson-PapapetrouTulczyjew-Dixon equations in ultra-relativistic regime and gravimagnetic moment

Desvignes, G., see Goddi

Devulder, C., see Caldwell

Di Virgilio, A. D. V., GINGER

Diab, A., see Tawfik

Disconzi, M. M., Kephart, T. W. \& Scherrer, R. J., On a viable first-order formulation of relativistic viscous fluids and its applications to cosmology

Do, T. Q. \& Nguyen, S. H. Q., Anisotropic power-law inflation in a two-scalarfield model with a mixed kinetic term

Doostmohammadi, S., Estimation of mass composition of high energy cosmic rays via modeling a relation between lateral distribution parameters and shower age

Dunsby, P. K. S., see Abebe

Dunsby, P. K. S., see Nzioki

Dunsby, P., see van de Bruck

Duston, C. L., Using cosmic strings to relate local geometry to spatial topology

Dzhunushaliev, V., Folomeev, V., Kleihaus, B. \& Kunz, J., Dispersion relations for gravitational waves in different models of dark energy

Dzhunushaliev, V., Folomeev, V., Kleihaus, B. \& Kunz, J., Extended objects in nonperturbative
26 (2017) 1741015

26 (2017) 1730001

26 (2017) 1742001

26 (2017) 1750086

26 (2017) 1750117

26 (2017) 1750047

26 (2017) 1730001

26 (2017) 1742005

26 (2017) 1741016

26 (2017) 1750166

26 (2017) 1750146

26 (2017) 1750157
26 (2017) 1750161

26 (2017) 1750054

26 (2017) 1750048

26 (2017) 1750152

26 (2017) 1750033 
quantum-field theory and the cosmological constant Easson, D. A., see Dent Eatough, R. P., see Goddi Ebrahimi, E., Golchin, H., Mehrabi, A. \& Movahed, S. M. S., Consistency of nonlinear interacting ghost dark energy with recent observations

Ebrahimi, E., see Golchin

Eingorn, M., Kiefer, C. \& Zhuk, A., Cosmic screening of the gravitational interaction

Eingorn, M., Cosmological law of universal gravitation

Eisenhauer, F., see Goddi

El Dahab, E. Abou, see Tawfik

El Hanafy, W. \& Nashed, G. G. L., Lorenz gauge fixing of $f(T)$ teleparallel cosmology

Engelhardt, N. \& Fischetti, S., Locality from quantum gravity: All or nothing

English, J., Canvas and cosmos: Visual art techniques applied to astronomy data

Escamilla-Rivera, C., see Castellanos

Eslam Panah, B., see Hendi

Estes, J., Kavic, M., Lippert, M. \& Simonetti, J. H., Pulsar-black hole binaries as a window on quantum gravity

Falcke, H., see Goddi

Falomo, R., see Scarpa

Farrugia, C. R. \& Sultana, J., The effects of spatial curvature on cosmic evolution

Felegary, F., see Setare

Fernando, S. \& Manning, A., Electromagnetic perturbations of a de Sitter black hole in massive gravity

Fernando, S., see Bretón

Fernando, S., Bardeen-de Sitter black holes

Feroze, T., see Jamil

Feroze, T., see Mahmood
26 (2017) 1750074

26 (2017) 1750117

26 (2017) 1730001

26 (2017) 1750124

26 (2017) 1750098

26 (2017) 1743012

26 (2017) 1750121

26 (2017) 1730001

26 (2017) 1750166

26 (2017) 1750154

26 (2017) 1743028

26 (2017) 1730010

26 (2017) 1750032

26 (2017) 1750026

26 (2017) 1743004

26 (2017) 1730001

26 (2017) 1750067

26 (2017) 1750115

26 (2017) 1750101

26 (2017) 1750100

26 (2017) 1750112

26 (2017) 1750071

26 (2017) 1741006

26 (2017) 1741008
Ferreira, R. Z., Sandora, M. \& Sloth, M. S., Is patience a virtue? Cosmic censorship of infrared effects in de Sitter

Filippi, S., see Rueda

Fischer, A. E., A simple alltime model for the birth, big bang, and death of the universe

26 (2017) 1743019

26 (2017) 1730016

Fischetti, S., see Engelhardt

Flachi, A. \& Fukushima, K., Astrophysical jets from topological currents

Folomeev, V., see Dzhunushaliev

Folomeev, V., see Dzhunushaliev

Fraga-Encinas, R., see Goddi

Francois, S., see Hansson

Freese, K., Status of dark matter in the universe

Fromm, C. M., see Goddi

$\mathrm{Fu}$, X. \& Li, P., Testing the distance-duality relation from strong gravitational lensing, type Ia supernovae and gammaray bursts data up to redshift $z \sim 3.6$

Fuksman, J. D. M., see Rueda

Fukushima, K., see Flachi

Gan, W.-C. \& Shu, F.-W., Holography as deep learning

Ganguly, C., see Barrow

Gayakwad, P. V., see Borkar

Gehrels, N. \& Cannizzo, J.

K., Explosions throughout the universe

George, M., see Bhatt

Ghayour, B., Further investigation about inflation and reheating stages based on the Planck and WMAP-9

Ghayour, B., The investigation of detectability of the relic gravitational waves based on the WMAP-9 and Planck

Gillessen, S., see Goddi

Giordano, M., Nucita, A. A., De Paolis, F. \& Ingrosso,
26 (2017) 1743014

26 (2017) 1743028

26 (2017) 1743007

26 (2017) 1750157

26 (2017) 1750074

26 (2017) 1730001

26 (2017) 1743003

26 (2017) 1730012

26 (2017) 1730001

26 (2017) 1750097

26 (2017) 1730016

26 (2017) 1743020

26 (2017) 1743016

26 (2017) 1750061

26 (2017) 1730003

26 (2017) 1750052

26 (2017) 1750079

26 (2017) 1750003

26 (2017) 1730001
26 (2017) 1743007 
G., Timing analysis in microlensing

Giordano, M., see Nucita

Goddi, C., Falcke, H., Kramer, M., Rezzolla, L., Brinkerink, C., Bronzwaer, T., Davelaar, J. R. J., Deane, R., De Laurentis, M., Desvignes, G., Eatough, R. P., Eisenhauer, F., FragaEncinas, R., Fromm, C. M., Gillessen, S., Grenzebach, A., Issaoun, S., Janßen, M., Konoplya, R., Krichbaum, T. P., Laing, R., Liu, K., Lu, R.-S., Mizuno, Y., Moscibrodzka, M., Müller, C., Olivares, H., Pfuhl, O., Porth, O., Roelofs, F., Ros, E., Schuster, K., Tilanus, R., Torne, P., van Bemmel, I., van Langevelde, H. J., Wex, N., Younsi, Z. \& Zhidenko, A., BlackHoleCam: Fundamental physics of the galactic center

Gogberashvili, M., Conformal $(2+4)$-braneworld

Golchin, H., Jamali, S. \& Ebrahimi, E., Interacting dark energy: Dynamical system analysis

Golchin, H., see Ebrahimi

Gong, J.-O., Multi-field inflation and cosmological perturbations

Gong, Y., see Saitou

Gong, Y., see Yi

Gopir, G., see Soleimani

Goswami, R., see Nzioki

Govender, M., Bogadi, R. S. \& Maharaj, S. D., The influence of an equationof-state during radiative collapse

Govender, M., see Bhar

Graber, V., Andersson, N. \& Hogg, M., Neutron stars in the laboratory

Graça, J. P. M., Salako, G. I. \& Bezerra, V. B., Quasinormal modes of a black
26 (2017) 1741009

26 (2017) 1741015

26 (2017) 1730001

26 (2017) 1750125

26 (2017) 1750098

26 (2017) 1750124

26 (2017) 1740003

26 (2017) 1750132

26 (2017) 1750005

26 (2017) 1750043

26 (2017) 1750048

26 (2017) 1750065

26 (2017) 1750053

26 (2017) 1730015 hole with a cloud of strings in Einstein-Gauss-Bonnet gravity

Greenwood, E., Quantum collapse of a charged $n$-dimensional BTZ-like domain wall

Grenzebach, A., see Goddi

Grigoryan, A. Kh., Mkrtchyan, A. R. \& Saharian, A. A., Fermionic condensate and the Casimir effect in cosmic string spacetime

Grøn, Ø., see Brevik

Gu, J.-A., Kim, S. P. \& Shen, C.-M., Gibbons-Hawking temperature corrected by quantum cosmology

Guendelman, E. I., see Benisty

Gulshan, F., see Jawad

Gulshan, F., see Jawad

Gupta, Y. K., see Maurya

Gurzadyan, A. V. \& Kocharyan, A. A., Superspace and global stability in general relativity

Gurzadyan, V. G., Ciufolini, I., Paolozzi, A., Kashin, A. L., Khachatryan, H. G., Mirzoyan, S. \& Sindoni, G., Satellites testing general relativity: Residuals versus perturbations

Ha, Y. K., Weighing the black holes of GW150914

Halilsoy, M., see Mazharimousavi

Hall, G., Some comparisons of the Weyl conformal tensor and its classification in 4-dimensional Lorentz, neutral or positive definite signatures

Hansraj, S., see Dadhich

Hansson, J. \& Francois, S., Testing quantum gravity

Hardwick, R. J., Vennin, V. \& Wands, D., A quantum window onto early inflation

Harko, T., see Belinchón

$\mathrm{He}$, P., see Sui
26 (2017) 1750113

26 (2017) 1750028

26 (2017) 1730001

26 (2017) 1750064

26 (2017) 1730024

26 (2017) 1750116

26 (2017) 1743021

26 (2017) 1750007

26 (2017) 1750049

26 (2017) 1750002

26 (2017) 1741019

26 (2017) 1741020

26 (2017) 1743018

26 (2017) 1750158

26 (2017) 1741004

26 (2017) 1750056

26 (2017) 1743003

26 (2017) 1743025

26 (2017) 1750073

26 (2017) 1750130 
Hendi, S. H., Panahiyan, S., Momennia, M. \& Eslam Panah, B., EinsteinGauss-Bonnet black holes with a perturbative nonlinear electrodynamics: Geometrical thermodynamics

Hesamifard, F. \& Rezaii, M. M., Evolution of the Robertson-Walker metric under 2-loop renormalization group flow

Heydari-Fard, M., Razmi, H. \& Yousefi, M., ScalarGauss-Bonnet gravity and cosmic acceleration: Comparison with quintessence dark energy

Hipólito-Ricaldi, W. S. \& Villanueva, J. R., A Jacobian generalization of the pseudo-NambuGoldstone boson potential

Hod, S., A proof of the weak gravity conjecture

Hofmann, W., Perspectives from CTA in relativistic astrophysics

Hogg, M., see Graber

Hong, B., see Zhou

Hou, D., see Yin

Houndjo, M. J. S., Rodrigues, M. E., Mazhari, N. S., Momeni, D. \& Myrzakulov, R., Higher-derivative $f(R, \square R, T)$ theories of gravity

Hsu, S. D. H., The measure problem in no-collapse (many worlds) quantum mechanics

Huang, H. \& Huang, L., The constraint of $H_{0}$ from galaxy clusters and Hubble parameter data

Huang, L., see Huang

Huang, Q.-G., Physics in the early universe

Huang, Y., Liu, D.-J. \& Li, X.-Z., Superradiant instability of $D$-dimensional Reissner-Nordström-antide Sitter black hole mirror system

Huang, Z., see Leung

Hussain, I., see Batool

26 (2017) 1750070

26 (2017) 1741005

26 (2017) 1750026

26 (2017) 1750021

26 (2017) 1750008

26 (2017) 1750019

26 (2017) 1742004

26 (2017) 1730005

26 (2017) 1730015

26 (2017) 1750077

26 (2017) 1750027

26 (2017) 1750024

26 (2017) 1730008

26 (2017) 1750129

26 (2017) 1750129

26 (2017) 1740001

26 (2017) 1750141
Hussain, T., Khurshudyan, M., Ahmed, S. \& Khurshudyan, As., Role of structure scalars on viscous and heat conducting spherical systems in $f(R, T)$ gravity

Ibrahim, Z. A., see Soleimani

Iftikhar, S., see Sharif

Ikram, A., see Sharif

Ikram, A., see Sharif

Ikram, A., see Sharif

Ilyas, A., see Jawad

Ilyas, A., see Jawad

Inan, N. A., A new approach to detecting gravitational waves via the coupling of gravity to the zeropoint energy of the phonon modes of a superconductor

Ingrosso, G., see Giordano

Ingrosso, G., see Nucita

Iqbal, Q., see Shah

Ismat Fatima, H., see Sharif

Issaoun, S., see Goddi

Jafarzade, Kh. \& Sadeghi, J., Phase transition and holographic in modified Horava-Lifshitz black hole

Jamali, S., see Golchin

Jamil, B. \& Feroze, T., Conservation laws corresponding to the Noether symmetries of the geodetic Lagrangian in spherically symmetric spacetimes

Jamil, M., see Majeed

Janßen, M., see Goddi

Jasim, M. K., see Maurya

Jawad, A. \& Umair Shahzad, M., Particle dynamics around time conformal regular black holes via Noether symmetries

Jawad, A., Azhar, N. \& Rani, S., Entropy corrected holographic dark energy models in modified gravity
26 (2017) 1750155

26 (2017) 1750043

26 (2017) 1750091

26 (2017) 1750030

26 (2017) 1750084

26 (2017) 1750104

26 (2017) 1750144

26 (2017) 1750031

26 (2017) 1743031

26(2017) 1741009

26 (2017) 1741015

26 (2017) 1750142

26 (2017) 1750109

26(2017) 1730001

26 (2017) 1750138

26 (2017) 1750098

26 (2017) 1741006

26 (2017) 1741017

26 (2017) 1730001

26 (2017) 1750002

26 (2017) 1750059

26 (2017) 1750040 
Jawad, A., Ilyas, A. \& Rani, S., Dynamics of modified Chaplygin gas inflation on the Brane with bulk viscous pressure

Jawad, A., Rani, S. \& Ilyas, A., Quartic potential inspired warm standard and tachyon scalar fields brane-world inflation

Jawad, A., Rani, S., Salako, I. G. \& Gulshan, F., Cosmological study in loop quantum cosmology through dark energy model

Jawad, A., Rani, S., Salako, I. G. \& Gulshan, F., Pilgrim dark energy models in fractal universe

Jeena, P. A., see Wahlang

Jetzer, P., General relativity tests with space clocks in highly elliptic orbits

Jia, H., see Zhou

Kachru, S. \& Tripathy, A., Black holes and Hurwitz class numbers

Kajuri, N., The time measurement problem in quantum cosmology

Kamali, V., Basilakos, S. \& Mehrabi, A., Measuring the effects of loop quantum cosmology in the CMB data

Karlica, M., see Rueda

Karlica, M., see Ruffini

Kashin, A. L., see Gurzadyan

Kausar, H. R., Polarization states of gravitational waves in modified theories

Kavic, M., see Estes

Kephart, T. W., see Dent

Kephart, T. W., see Disconzi

Khachatryan, H. G., see Gurzadyan

Khurshudyan, As., see Hussain

Khurshudyan, M., see Hussain

Kiefer, C., see Eingorn
$26(2017) 1750031$

26 (2017) 1750144

26 (2017) 1750007

26 (2017) 1750049

26 (2017) 1750160

26 (2017) 1741014

26 (2017) 1750077

26 (2017) 1742003

26 (2017) 1743011

26 (2017) 1743023

26 (2017) 1730016

26 (2017) 1730019

26 (2017) 1741020

26 (2017) 1741010

26 (2017) 1743004

26 (2017) 1750117

26 (2017) 1750146

26 (2017) 1741020

26 (2017) 1750155

26 (2017) 1750155

26 (2017) 1743012
Kiess, T. E., A resolution of a metric singularity associated with the introduction of $\Lambda$ into static spherically symmetric systems

Kileba Matondo, D., see Maharaj

Kim, K. Y., see Qadir

Kim, S. P., see Gu

Kim, W., The effective Tolman temperature in curved spacetimes

Kirillov, A. A. \& Savelova, E. P., Modification of gravity by a spherically symmetric wormhole

Kirillov, A. A., see Belotsky

Kleihaus, B., see Dzhunushaliev

Kleihaus, B., see Dzhunushaliev

Klinkhamer, F. R., A generalization of unimodular gravity with vacuummatter energy exchange

Kobakhidze, A., Lagger, C. \& Manning, A., Probing the quantum fuzziness of space and time with gravitational waves

Kobakhidze, A., Manning, A. \& Yue, J., Gravitational waves from the phase transition of a nonlinearly realized electroweak gauge symmetry

Kocharyan, A. A., see Gurzadyan

Konoplya, R., see Goddi

Korzyński, M., see Bolejko

Kosasih, J. S., see Ming

Kovacevic, M., see Rueda

Kovacevic, M., see Ruffini

Kramer, M., see Goddi

Krichbaum, T. P., see Goddi

Kruglov, S. I., Black hole solution in the framework of arctan-electrodynamics

Kuhfittig, P. K. F., Stable phantom-energy wormholes admitting conformal motions

Kunkolienkar, R. S. \& Banerjee, K., Towards
26 (2017) 1750039

26(2017) 1750014

26 (2017) 1741001

26 (2017) 1750116

26 (2017) 1730025

26 (2017) 1750145

26 (2017) 1750102

26 (2017) 1750157

26 (2017) 1750074

26 (2017) 1750006

26 (2017) 1743005

26 (2017) 1750114

26(2017) 1741019

26 (2017) 1730001

26 (2017) 1730011

26 (2017) 1750092

26 (2017) 1730016

26 (2017) 1730019

26 (2017) 1730001

26 (2017) 1730001

26 (2017) 1750075

26 (2017) 1750025 
a dS/MERA correspondence

Kunz, J., see Dzhunushaliev

Kunz, J., see Dzhunushaliev

Kuriakose, V. C., see Prasia

Lagger, C., see Kobakhidze

Laing, R., see Goddi

Lambiase, G., see Chakravarty

Lämmerzahl, C., see Castellanos

Lebed, A. G., Inequivalence between gravitational mass and energy due to quantum effects at microscopic and macroscopic levels

Lee, H. W., see Qadir

Lenka, S. S., Char, P. \& Banik, S., Critical mass, moment of inertia and universal relations of rapidly rotating neutron stars with exotic matter

Leung, J. S.-Y. \& Huang, Z., Marginalized Fisher forecast for Horndeski dark energy models

Li, H., see Yang

Li, H.-F., see Ma

Li, M., Some topics on scaleinvariant perturbations from noninflationary universe

Li, P., see Fu

Li, X.-Z., see Huang

Li, Y., Dynamics of twoscalar-field cosmological models beyond the exponential potential

Lima, J. A. S., see Tamayo

Lima, R. C., see Pereira

Lippert, M., see Estes

Liu, D.-J., see Huang

Liu, G., see Peng

Liu, K., see Goddi

Liu, Y.-S., see Ma

Logoteta, D., see Bombaci

López-Corredoira, M., see Melia

Loret, N., Meljanac, S., Mercati, F. \& Pikutić, D., Vectorlike deformations of relativistic quantum phase-space and relativistic kinematics

Loret, N., see AmelinoCamelia

Lu, J., see Yang

Lu, R.-S., see Goddi

Luongo, O., Pisani, G. B. \& Troisi, A., Cosmological degeneracy versus cosmography: A cosmographic dark energy model

Ma, M.-S., Liu, Y.-S. \& Li, H.-F., Revisit on the thermodynamic stability of the noncommutative Schwarzschild black hole

26 (2017) 1730022 26 (2017) 1741001

Ma, P., see Peng

Macías, A., see Castellanos

Mafa Takisa, P., see Maharaj

Maharaj, S. D., Kileba Matondo, D. \& Mafa Takisa, P., A family of Finch and Skea relativistic stars

26 (2017) 1750127

Maharaj, S. D., see Govender

26 (2017) $1750070 \quad$ A. \& Feroze, T., A class of

26 (2017) 1750013

26 (2017) 1750018

26 (2017) 1740005

26 (2017) 1750097

26 (2017) 1750141

26 (2017) 1750164

26 (2017) 1750093

26 (2017) 1730028

26 (2017) 1743004

26 (2017) 1750141

26 (2017) 1750046

26 (2017) 1730001

26 (2017) 1750018

26 (2017) 1730004

26 (2017) 1750055 nonstatic exact solutions of the Einstein-Maxwell field equations

Majeed, B. \& Jamil, M., Dynamics and center of mass energy of colliding particles around black hole in $f(R)$ gravity

Mak, M. K., see Belinchón

Maksimova, N. A., see Caldwell

Mamon, A. A., Reconstruction of interaction rate in holographic dark energy model with Hubble horizon as the infrared cut-off

Mancini, L., On the relationship between the planetary radius and the equilibrium temperature for transiting exoplanets

Mann, R. B., see Baccetti

Manna, T., see Bhar

Mannheim, P. D., Is the cosmological constant problem properly posed?
26 (2017) 1750123

26 (2017) 1750076

26 (2017) 1750013

26 (2017) 1730001

26 (2017) 1750015

26 (2017) 1750018

26 (2017) 1750046

26 (2017) 1750032

26(2017) 1750014

26 (2017) 1750014

26 (2017) 1750065

26 (2017) 1741008

26 (2017) 1741017

26 (2017) 1750073

26 (2017) 1742005

26 (2017) 1750136

26 (2017) 1741012

26 (2017) 1743008

26 (2017) 1750090

26 (2017) 1743009 
Manni, L., see Nucita

Manning, A., see Fernando

Manning, A., see Kobakhidze

Manning, A., see Kobakhidze

Mansoori, S. A. H., see Sherkatghanad

Manzoor, R., see Sharif

Marciu, M., Dynamical properties of scaling solutions in teleparallel dark energy cosmologies with nonminimal coupling

Marongwe, S., The electromagnetic signature of gravitational wave interaction with the quantum vacuum

Martín-Ruiz, A. \& Urrutia, L. F., Gravitational waves propagation in nondynamical Chern-Simons gravity

Masi, N. \& Ballardini, M., A conservative assessment of the current constraints on dark matter annihilation from cosmic rays and CMB observations

Masi, N., Rényi entropy for particle systems as an instrument to enlarge the Boltzmannian concept of entropy: Some holographic perspectives

Mathur, S. D., Spacetime has a "thickness"

Maurya, S. K., Gupta, Y. K., Dayanandan, B., Jasim, M. K. \& Al-Jamel, A., Relativistic anisotropic models for compact star with equation of state $p=$ $f(\rho)$

Mavromatos, N. E., Argüelles, C. R., Ruffini, R. \& Rueda, J. A., Selfinteracting dark matter

Mazhari, N. S., see Houndjo

Mazharimousavi, S. H., Halilsoy, M. \& Amen, S. N. H., Stability of spherically symmetric timelike thin-shells in general
26 (2017) 1741015

26 (2017) 1750100

26 (2017) 1750114

26 (2017) 1743005

26 (2017) 1750017

26 (2017) 1750057

26 (2017) 1750103

26 (2017) 1750020

26 (2017) 1750148

26 (2017) 1750041

26 (2017) 1750105

26 (2017) 1742002

26 (2017) 1750002

26 (2017) 1730007

26 (2017) 1750024 relativity with a variable equation-of-state

26 (2017) 1750158

26 (2017) 1750044

26 (2017) 1750124

26 (2017) 1743023

Mehrabi, A., see Kamali

Melia, F. \& LópezCorredoira, M., AlcockPaczyński test with model-independent BAO data

Meljanac, S., see Loret

Melon Fuksman, J. D., see Ruffini

Merafina, M., Dynamical evolution of globular clusters: Recent developments

Mercati, F., see Loret

Mezcua, M., Observational evidence for intermediatemass black holes

Ming, K., Triyanta \& Kosasih, J. S., Gravitoelectromagnetism in teleparallel equivalent of general relativity: A new alternative

Mirza, B., see Sherkatghanad

Mirzaiyan, Z., see Sherkatghanad

Mirzoyan, S., see Gurzadyan

Mizuno, Y., see Goddi

Mkrtchyan, A. R., see Grigoryan

Modesto, L. \& Rachwał, L., Nonlocal quantum gravity: A review

Mohanty, S., see Chakravarty

Momeni, D., see Houndjo

Momennia, M., see Hendi

Monerat, G. A., Corrêa Silva, E. V., Neves, C., Oliveira-Neto, G., Rezende Rodrigues, L. G. \& Silva de Oliveira, M., Can noncommutativity affect the whole history of the universe?

Monerat, G. A., see OliveiraNeto

Moradi, R., see Rueda

Moradi, R., see Ruffini

Moradpour, H., see Zadeh

Mori, M., see Tsuchida
26 (2017) 1750055

26 (2017) 1750123

26 (2017) 1730019

26 (2017) 1730017

26 (2017) 1750123

26 (2017) 1730021

26 (2017) 1750092

26(2017) 1750017

26(2017) 1750017

26 (2017) 1741020

26 (2017) 1730001

26 (2017) 1750064

26 (2017) 1730020

26 (2017) 1730023

26 (2017) 1750024

26(2017) 1750026

26 (2017) 1750022

26 (2017) 1750011

26 (2017) 1730016

26 (2017) 1730019

26 (2017) 1750080

26 (2017) 1750095 
Morley, P. D. \& Buettner, D. J., Dark matter in the local group of galaxies

Moscibrodzka, M., see Goddi

Movahed, S. M. S., see Ebrahimi

Mu, X., see Zhou

Müller, C., see Goddi

Muccino, M., see Rueda

Muccino, M., see Ruffini

Mullick, L., see Chakravarty

Mumtaz, S., see Sharif

Myrzakulov, R., see Houndjo

Naji, J., see Pourhassan

Namjoo, M. H., see Chen

Nashed, G. G. L., see El Hanafy

Navarro-Salas, J., see Agullo

Nazarova, N. O., see Belotsky

Nelson, E. \& Riedel, C. J., Classical entanglement structure in the wavefunction of inflationary fluctuations

Neves, C., see Monerat

Nguyen, S. H. Q., see Do

Noureen, I. \& Zubair, M., Axially symmetric shearfree fluids in $f(R, T)$ gravity

Nozari, K. \& Ramezani, H., Thermodynamical dimensional reduction in a UV \& IR-deformed Snyder space

Nozari, K. \& Rashidi, N., Some aspects of nonminimal inflation driven by a superpotential

Nozari, K. \& Shafizadeh, S., Cosmological inflation in a generalized unimodular gravity

Nozari, K. \& Shafizadeh, S., Realization of blue spectrum in generalized Galileon super-inflation models

Nucita, A. A., De Paolis, F., Ingrosso, G., Giordano, M. \& Manni, L., Astrometric microlensing
Nucita, A. A., see Giordano

Nzioki, A. M., Goswami, R. \& Dunsby, P. K. S., Covariant perturbations of Schwarzschild black holes in $f(R)$ gravity

26 (2017) 1750124

26 (2017) 1750077

26 (2017) 1730001

26 (2017) 1730016

26 (2017) 1730019

26 (2017) 1750122

26 (2017) 1741007

26 (2017) 1750024

26 (2017) 1750012

26 (2017) 1740004

26 (2017) 1750154

26 (2017) 1742001

26 (2017) 1750102

26 (2017) 1743006

26 (2017) 1750022

26 (2017) 1750072

26 (2017) 1750128

26 (2017) 1750126

26 (2017) 1750058

26 (2017) 1750107

26 (2017) 1750016

26 (2017) 1741015 gravity

Odintsov, S. D. \& Oikonomou, V. K., Big bounce with finite-time singularity: The $F(R)$ gravity description

Odintsov, S. D., see Brevik

Oikonomou, V. K., see Odintsov

Oikonomou, V. K., Inflation and bounce from classical and loop quantum cosmology imperfect fluids

Olivares, H., see Goddi

Oliveira-Neto, G., Silva de Oliveira, M., Monerat, G. A. \& Corrêa Silva, E. V., Noncommutativity in the early universe

Oliveira-Neto, G., see Monerat

Osmanov, Z., see Arsenadze

Osmanov, Z., see Bakhtadze

Osmanov, Z., New mechanism of acceleration of particles by stellar black holes

Otoya, V. J. V., see Assimos

Ottolina, R., see Scarpa

Owaidat, M. Q., see AlBadawi

Padmanabhan, H., see Padmanabhan

Padmanabhan, T. \& Padmanabhan, H., Quantum gravity at Hubble scales determines the cosmological constant and the amplitude of primordial perturbations

Paduraru, L. E., see van de Bruck

Paik, B., Test of indestructibility of a nonsingular black hole

Panahiyan, S., see Hendi

Panotopoulos, G., see Videla Pant, N., see Bhar

\section{6 (2017) 1741009}

26 (2017) 1750048

26 (2017) 1750023
26 (2017) 1750085

26 (2017) 1730024

$26(2017) 1750085$

26 (2017) 1750110

26 (2017) 1730001

26 (2017) 1750011

26 (2017) 1750022

26 (2017) 1750153

26 (2017) 1750135

26 (2017) 1750034

26 (2017) 1750087

26 (2017) 1750067

26 (2017) 1750169

26 (2017) 1743002

26 (2017) 1743002

26 (2017) 1750152

26 (2017) 1750165

26 (2017) 1750026

26 (2017) 1750066

26 (2017) 1750078 
Paolozzi, A., see Gurzadyan

Papini, G., Classical and quantum aspects of particle propagation in external gravitational fields

Pazhouhesh, R., see Roobiat

Pe'er, A. \& Ryde, F., Photospheric emission in gamma-ray bursts

Pedram, P., see Shababi

Penacchioni, A. V., see Rueda

Penacchioni, A. V., see Ruffini

Peng, Y., Chen, T., Liu, G. \& Ma, P., Various types of holographic metal/superconductor phase transitions with dark matter sector

Pereira, A. D., see Assimos

Pereira, S. H. \& Lima, R. C., Creation of mass dimension one fermionic particles in asymptotically expanding universe

Pfuhl, O., see Goddi

Pikutić, D., see Loret

Pincak, R., see Sepehri

Pisani, G. B., see Luongo

Pisani, G. B., see Rueda

Pisani, G. B., see Ruffini

Pollock, M. D., On the internal state of the Schwarzschild black hole

Porth, O., see Goddi

Pourhassan, B. \& Naji, J., Tachyonic matter cosmology with exponential and hyperbolic potentials

Pradhan, P., Thermodynamic products in extended phase-space

Prasia, P. \& Kuriakose, V. C., Data analysis of massive gravitational waves from gamma ray bursts

Primorac, D., see Rueda

Primorac, D., see Ruffini

Pshirkov, M. S., May axion clusters be sources of fast radio bursts?

Qadir, A., Lee, H. W. \& Kim, K. Y., Modified relativistic dynamics
26 (2017) 1741020

26 (2017) 1750137

26 (2017) 1750134

26 (2017) 1730018

26 (2017) 1750081

26 (2017) 1730016

26 (2017) 1730019

26 (2017) 1750046

26 (2017) 1750087

26 (2017) 1730028

26 (2017) 1730001

26 (2017) 1750123

26 (2017) 1750094

26 (2017) 1750015

26 (2017) 1730016

26 (2017) 1730019

26 (2017) 1750088 26 (2017) 1730001

26 (2017) 1750012

26 (2017) 1750010

26 (2017) 1750150

26 (2017) 1730016

26 (2017) 1730019

26 (2017) 1750068

26 (2017) 1741001
Qadir, A., see Usman

Qadir, A., Revisiting the energy in gravitational waves

Quintanar, G. L. \& de la Macorra, A., Cosmological implications of Nambu-Jona-Lasinio model with a dynamical coupling

Rachwał, L., see Modesto

Radiman, S., see Soleimani

Rafaqat, A., see Yousaf

Rahaman, F., see Bhar

Rajan, D. \& Visser, M., Cartesian Kerr-Schild variation on the NewmanJanis trick

Ramezani, H., see Nozari

Ramírez, W. G., see Deriglazov

Rani, S., see Jawad

Rani, S., see Jawad

Rani, S., see Jawad

Rani, S., see Jawad

Rani, S., see Jawad

Rashidi, N., see Nozari

Razmi, H., see Heydari-Fard

Rezaii, M. M., see Hesamifard

Rezende Rodrigues, L. G., see Monerat

Rezzolla, L., see Goddi

Riedel, C. J., see Nelson

Rizwan, M. \& Saifullah, K., GUP-corrected thermodynamics of accelerating and rotating black holes

Rodrigues, E. L., see Barreto

Rodrigues, M. E., see Houndjo

Rodriguez-Mueller, B., see Barreto

Roelofs, F., see Goddi

Roobiat, K. Y. \& Pazhouhesh, R., Reconstruction of $\Lambda \mathrm{CDM}$ expansion in nonlocal gravity

Ros, E., see Goddi

Rosati, G., see AmelinoCamelia

Rubin, S. G., see Belotsky

Rueda, J. A., Aimuratov, Y., de Almeida, U. B., Becerra, L., Bianco, C.
26 (2017) 1741003

26 (2017) 1741002

26 (2017) 1750004

26 (2017) 1730020

26 (2017) 1750043

26 (2017) 1750099

26 (2017) 1750078

26 (2017) 1750167

26 (2017) 1750126

26 (2017) 1750047

26 (2017) 1750144

26(2017) 1750007

26 (2017) 1750031

26 (2017) 1750040

26 (2017) 1750049

26 (2017) 1750058

26 (2017) 1750008

26 (2017) 1750021

26 (2017) 1750022

26(2017) 1730001

26 (2017) 1743006

26 (2017) 1741018

26(2017) 1743017

26 (2017) 1750024

26(2017) 1750096

26 (2017) 1730001

26 (2017) 1750134

26 (2017) 1730001

26 (2017) 1750076

26 (2017) 1750102 
L., Cherubini, C., Filippi, S., Karlica, M., Kovacevic, M., Fuksman, J. D. M., Moradi, R., Muccino, M., Penacchioni, A. V., Pisani, G. B., Primorac, D., Ruffini, R., Sahakyan, N., Shakeri, S. \& Wang, Y., The binary systems associated with short and long gamma-ray bursts and their detectability

Rueda, J. A., see Mavromatos

Rueda, J. A., see Ruffini

Ruffini, R., Aimuratov, Y., Becerra, L., Bianco, C. L., Karlica, M., Kovacevic, M., Melon Fuksman, J. D., Moradi, R., Muccino, M., Penacchioni, A. V., Pisani, G. B., Primorac, D., Rueda, J. A., Shakeri, S., Vereshchagin, G. V., Wang, Y. \& Xue, S.S., The cosmic matrix in the 50th anniversary of relativistic astrophysics

Ruffini, R., see Mavromatos

Ruffini, R., see Rueda

Ryde, F., see Pe'er

Sachan, S., see Singh

Sadeghi, J., see Jafarzade

Sadeghian, S. \& Shafiekhani, A., Extremal charged Vaidya and its near horizon geometry

Sahakyan, N., see Rueda

Saharian, A. A., see Grigoryan

Saifullah, K., see Rizwan

Saitou, R. \& Gong, Y., de Sitter spacetime with a Becchi-Rouet-Stora quartet

Salako, G. I., see Graça

Salako, I. G., see Jawad

Salako, I. G., see Jawad

Sandora, M., see Ferreira

Santos, T. R. S., see Assimos

Sanyal, A. K., see Sk

Saridakis, E. N., see Brevik

Saridakis, E. N., see Sepehri

Sartori, G., see Conti

Savelova, E. P., see Kirillov

Sawado, N., see Brihaye

Scarpa, R., Ottolina, R., Falomo, R. \& Treves, A., Dynamics of wide binary stars: A case study for testing Newtonian dynamics in the low acceleration regime

Schee, J., see Abdujabbarov

Scherrer, R. J., see Disconzi

Schober, G. A. H., see Starke

26 (2017) 1730016

26 (2017) 1730007

26 (2017) 1730019

26 (2017) 1730019

26 (2017) 1730007

26 (2017) 1730016

26 (2017) 1730018

26 (2017) 1750038

26 (2017) 1750138

26 (2017) 1750036

26 (2017) 1730016

26 (2017) 1750064

26 (2017) 1741018

26 (2017) 1750132

26 (2017) 1750113

26 (2017) 1750007

26 (2017) 1750049

26 (2017) 1743019

26 (2017) 1750087

26 (2017) 1750162

26 (2017) 1730024

26 (2017) 1750094

26 (2017) 1750083

26 (2017) 1750145
Schuster, K., see Goddi

Sefiedgar, A. S. \& Daghigh, M., Thermodynamics of the FRW universe in rainbow gravity

Sepehri, A., Pincak, R., Bamba, K., Capozziello, S. \& Saridakis, E. N., Current density and conductivity through modified gravity in the graphene with defects

Setare, M. R., Felegary, F. \& Darabi, F., Evolution of spherical overdensities in new agegraphic dark energy model

Shababi, H. \& Pedram, P., Hořava-Lifshitz quantum cosmology in the presence of Chaplygin gas: Exact solutions and the late-time acceleration

Shabani, H., Cosmological consequences and statefinder diagnosis of a noninteracting generalized Chaplygin gas in $f(R, T)$ gravity

Shafiekhani, A., see Sadeghian

Shafizadeh, S., see Nozari

Shafizadeh, S., see Nozari

Shah, H. H. \& Iqbal, Q., Gravitational collapse of dark matter interacting with dark energy: Black hole formation

Shaker, F., see Sheykhi

Shakeri, S., see Rueda

Shakeri, S., see Ruffini

Sharif, M. \& Iftikhar, S., Effects of electromagnetic field on the motion
26 (2017) 1750086

26 (2017) 1750067

26 (2017) 1741011

26 (2017) 1750146

26 (2017) 1750163

26 (2017) 1730001

26 (2017) 1750139

26 (2017) 1750094

26 (2017) 1750101

26 (2017) 1750081

26 (2017) 1750120

26 (2017) 1750036

26 (2017) 1750107

26 (2017) 1750016

26 (2017) 1750142

26 (2017) 1750050

26 (2017) 1730016

26 (2017) 1730019 
of particles in dyonic Reissner-Nordström black hole

Sharif, M. \& Ikram, A., Inflationary dynamics in $f(\mathcal{G})$ gravity

Sharif, M. \& Ikram, A., Instability analysis of expansion-free sphere in $f(\mathcal{G})$ gravity

Sharif, M. \& Ikram, A., Stability analysis of Einstein universe in $f(\mathcal{G}, T)$ gravity

Sharif, M. \& Ismat Fatima, H., Evolution of axially symmetric systems and $f(G)$ gravity

Sharif, M. \& Manzoor, R., Dark energy and collapsing axial system

Sharif, M. \& Mumtaz, S., Dynamics of thin-shell wormholes with different cosmological models

Shen, C.-M., see Gu

Sherkatghanad, Z., Mirza, B., Mirzaiyan, Z. \& Mansoori, S. A. H., Critical behaviors and phase transitions of black holes in higher order gravities and extended phase spaces

Sheykhi, A. \& Shaker, F., Effects of backreaction and exponential nonlinear electrodynamics on the holographic superconductors

Sheykhi, A., see Zadeh

Shu, F.-W., see Gan

Siddiqui, A. A., see Mahmood

Silva de Oliveira, M., see Monerat

Silva de Oliveira, M., see Oliveira-Neto

Simonetti, J. H., see Estes

Sindoni, G., see Gurzadyan

Singh, A., see Bao

Singh, D. V. \& Sachan, S., Logarithmic corrections to the entropy of scalar field in BTZ black hole spacetime
Singh, D. V., see Chaturvedi

Singh, K. N., see Bhar

26 (2017) 1750091

Singh, Ksh. N., see Bhar

Singh, N. K., see Chaturvedi

Singh, T. P., A new length

26 (2017) 1750030 scale for quantum gravity: A resolution of the black hole information loss paradox

26 (2017) 1750104

Sivaram, C., Dark matter (energy) may be indistinguishable from modified gravity (MOND)

26 (2017) 1750084

26 (2017) 1750109

26 (2017) 1750057

26 (2017) 1741007

26 (2017) 1750116

26 (2017) 1750017

26 (2017) 1750050

26 (2017) 1750080

26 (2017) 1743020

26 (2017) 1741008

26 (2017) 1750022

26 (2017) 1750011

26 (2017) 1743004

26 (2017) 1741020

26 (2017) 1743013

26 (2017) 1750038

Sk, N. \& Sanyal, A. K., Why scalar-tensor equivalent theories are not physically equivalent?

Sloth, M. S., see Ferreira

Smailagic, A., see Spallucci

Sobreiro, R. F., see Assimos

Soda, J., see Aoki

Soleimani, M. J., Abbasvandi, N., Gopir, G., Ibrahim, Z. A., Radiman, S. \& Wan Abdullah, W. A. T., Natural cutoffs effect on charged rotating $\mathrm{TeV}$ scale black hole thermodynamics

Solomons, D., see Abebe

Spallucci, E. \& Smailagic, A., Regular black holes from semi-classical down to Planckian size

Starke, R. \& Schober, G. A. H., Covariant response theory and the boost transform of the dielectric tensor

Stuchlík, Z., see Abdujabbarov

Stuchlík, Z., see Abdujabbarov

Stuchlík, Z., see Cremaschini

Sui, N. \& He, P., Time evolution of entropy for spherical self-gravitating systems

Sultana, J., see Farrugia

Sun, Y., see Xu

Sur, S., see Bhatia

Tamayo, D. A., Lima, J. A. S. \& Bessada, D. F. A., Production of primordial
26 (2017) 1750082

26 (2017) 1750090

26 (2017) 1750078

26(2017) 1750082

26 (2017) 1743015

26 (2017) 1743010
26 (2017) 1750043

26 (2017) 1750054

26 (2017) 1730013

26 (2017) 1750163

26 (2017) 1741011

26 (2017) 1750051

26 (2017) 1750001

26 (2017) 1750162

26 (2017) 1743019

26 (2017) 1730013

26 (2017) 1750087

26 (2017) 1750063

$\mathbf{2 6}(2017) 1750163$
$\mathbf{2 6}(2017) 1741011$
$\mathbf{2 6}(2017) 1750051$

26 (2017) 1750130

26 (2017) 1750115

26 (2017) 1750151

26 (2017) 1750149 
gravitational waves in a simple class of running vacuum cosmologies

Tarawneh, S., see Al-Badawi

Tawfik, A., Diab, A. \& El Dahab, E. Abou, Minimalsupersymmetric extended inflation field in HoravaLifshitz gravity

Terno, D. R., see Baccetti

't Hooft, G., Local conformal symmetry in black holes, standard model, and quantum gravity

Tilanus, R., see Goddi

Tomaz, A. A., see Assimos

Torne, P., see Goddi

Toshmatov, B., see Abdujabbarov

Toshmatov, B., see Abdujabbarov

Treves, A., see Scarpa

Tripathy, A., see Kachru

Triyanta, see Ming

Troisi, A., see Luongo

Tsuchida, S. \& Mori, M., The electron plus positron spectrum from annihilation of Kaluza-Klein dark matter in the Galaxy

Umair Shahzad, M., see Jawad

Urrutia, L. F., see MartínRuiz

Usman, M. \& Qadir, A., The extra scalar degrees of freedom from the twoHiggs doublet model for dark energy

van Bemmel, I., see Goddi

van de Bruck, C., Dunsby, P. \& Paduraru, L. E., Reheating and preheating in the simplest extension of Starobinsky inflation

van Langevelde, H. J., see Goddi

Vennin, V., see Hardwick

Vereshchagin, G. V., see Ruffini

Vetrugno, D., LISA pathfinder first results

Viaggiu, S., A possible new cosmological redshift

effect due to $\Lambda$ on traveling gravitational waves in Friedmann universes

26 (2017) 1750093 26 (2017) 1750169

26 (2017) 1750166 26 (2017) 1743008

$\mathbf{2 6}(2017) 1730006$
$\mathbf{2 6}(2017) 1730001$
$\mathbf{2 6}(2017) 1750087$
$\mathbf{2 6}(2017) 1730001$

26 (2017) 1741011

26 (2017) 1750051

26 (2017) 1750067

26 (2017) 1742003

26 (2017) 1750092

26(2017) 1750015

26 (2017) 1750095

26 (2017) 1750059

26 (2017) 1750148

26 (2017) 1741003

26 (2017) 1730001

26 (2017) 1750152

26 (2017) 1730001

26 (2017) 1743025

26 (2017) 1730019

26 (2017) 1741023
Videla, N. \& Panotopoulos, G., Natural Inflation on the brane with a TeV-scale gravity: Parameter constraints after Planck 2015

Vieira, H. S., Resonant frequencies of the hydrodynamic vortex

Villanueva, J. R., see Hipólito-Ricaldi

Visser, M., see Rajan

Visser, M., Quantum mechanix plus Newtonian gravity violates the universality of free fall

Wahlang, W., Jeena, P. A. \& Chakrabarti, S., Quasinormal modes of scalar and Dirac perturbations of Bardeen de Sitter black holes

Wan Abdullah, W. A. T., see Soleimani

Wan, Y., see Brandenberger

Wands, D., see Hardwick

Wang, A., Hořava gravity at a Lifshitz point: A progress report

Wang, H., see Zhou

Wang, Y., see Chen

Wang, Y., see Rueda

Wang, Y., see Ruffini

Wei, J.-J. \& Wu, X.-F., Gamma-ray burst cosmology: Hubble diagram and star formation history

Wen, W.-Y., Thermodynamic metric of deformed Schwarzschild black holes

Wex, N., see Goddi

White, S. C., see Dent

Wong, P. J., Shape dynamical loop gravity from a conformal Immirzi parameter

$\mathrm{Wu}, \mathrm{C}$., Scalar field quasinormal frequencies of Reissner-Nordström black hole surrounded by quintessence by using the continued fraction method
26 (2017) 1750168

26 (2017) 1750066

26 (2017) 1750035

26 (2017) 1750019

26 (2017) 1750167

26 (2017) 1743027

26 (2017) 1750160

26 (2017) 1750043

26 (2017) 1740006

26 (2017) 1743025

26 (2017) 1730014

26 (2017) 1750077

26 (2017) 1740004

26 (2017) 1730016

26 (2017) 1730019

26 (2017) 1730002

26 (2017) 1750106

26 (2017) 1730001

26 (2017) 1750117

26 (2017) 1750131

26 (2017) 1750111 
Wu, X.-F., see Wei

Wu, Y., see Yang

$\mathrm{Xu}, \quad \mathrm{H} . \quad \& \quad \mathrm{Xu}, \quad \mathrm{Z} .-\mathrm{M}$., Maxwell's equal area law for Lovelock thermodynamics

$\mathrm{Xu}$, H., Sun, Y. \& Zhao, L., Black hole thermodynamics and heat engines in conformal gravity

Xu, Z.-M., see Xu

Xue, S.-S., see Ruffini

Yahalom, A., Gravity, stability and cosmological models

Yang, W., Li, H., Wu, Y. \& Lu, J., Cosmological implications of the dark matter equation of state

Yi, Z. \& Gong, Y., PPN parameters in gravitational theory with nonminimally derivative coupling

Yin, L. \& Hou, D., s-Wave holographic superconductor in different ensembles and its thermodynamic consistency

Yoshii, H., see Brihaye

Younsi, Z., see Goddi

Yousaf, Z., Zaeem-ul-Haq Bhatti, M. \& Rafaqat, A., LTB geometry with tilted and nontilted congruences in $f(R, T)$ gravity

Yousaf, Z., see Bhatti

Yousaf, Z., see Zaeem-ulHaq Bhatti

Yousefi, M., see HeydariFard

Yue, J., see Kobakhidze
26 (2017) 1730002 26 (2017) 1750013

26 (2017) 1750037

26 (2017) 1750151

26 (2017) 1750037

26 (2017) 1730019

26 (2017) 1743026

26 (2017) 1750013

26 (2017) 1750005

26 (2017) 1750027

26 (2017) 1750086

26 (2017) 1730001

26 (2017) 1750099 26 (2017) 1750045

26 (2017) 1750029

26 (2017) 1750008

26 (2017) 1750114
Zadeh, M. A., Sheykhi, A. \& Moradpour, H., Holographic dark energy with the sign-changeable interaction term

26 (2017) 1750080

Zaeem-ul-Haq Bhatti, M. \& Yousaf, Z., Dynamical variables and evolution of the universe

Zaeem-ul-Haq Bhatti, M., see Yousaf

Zaslavskii, O. B., Black hole with a scalar field as a particle accelerator

Zaslavskii, O. B., SuperPenrose process due to collisions inside ergosphere

26 (2017) 1750009

Zhang, X., see Brandenberger

26 (2017) 1740006

26 (2017) 1750151

Zhao, L., see Xu

Zhao, X.-F., Property difference between the neutron star PSR J0348+0432 and its proto neutron star

Zhidenko, A., see Goddi

Zhou, S. \& Chen, G.-R., Corrected black hole thermodynamics in DamourRuffini's method with generalized uncertainty principle

Zhou, X., Jia, H., Hong, B., Mu, X. \& Wang, H., Effects of trapped neutrinos on the composition and structure of massive protoneutron stars Zhuk, A., see Eingorn Zubair, M., see Noureen
26 (2017) 1750062

26 (2017) 1750077 26 (2017) 1743012

26 (2017) 1750133

26 (2017) 1730001

26 (2017) 1750128 